\title{
Spatiotemporal distortions of attosecond pulses
}

\author{
Charles Bourassin-Bouchet,* Sébastien de Rossi, Franck Delmotte, and Pierre Chavel \\ Laboratoire Charles Fabry, Institut d'Optique, Université Paris-Sud, CNRS, \\ Campus Polytechnique, RD128, 91127 Palaiseau CEDEX, France \\ *Corresponding author: charles.bourassin-bouchet@institutoptique.fr
}

Received January 29, 2010; revised April 20, 2010; accepted April 26, 2010; posted April 27, 2010 (Doc. ID 123496); published May 19, 2010

\begin{abstract}
In this article, we report that usual multilayer mirror configurations to focus attosecond pulses generate geometric aberrations and can significantly stretch pulses. The numerical simulations show that the effects can be strong enough to delay some parts of the pulses of an attosecond pulse train and make them interfere with the next pulses of the train. The influence of the numerical aperture on the pulse duration is also studied, showing that such effects can occur even with very low numerical apertures. () 2010 Optical Society of America
\end{abstract}

OCIS codes: $320.0320,340.7480,080.1010$.

\section{INTRODUCTION}

For a decade, it has been possible to generate attosecond pulses. Such pulses are produced by the interaction of an infrared femtosecond pulse with a rare gas jet [1,2], a molecular gas jet [3], or even a solid target [4], which generates high harmonics of the fundamental infrared frequency in the extreme ultraviolet (EUV) spectral range. This is equivalent to a chirped pulse train in time $[5,6]$. But it is also possible to generate single attosecond pulses [7]. The current pulse duration record is 80 as [8] and has been obtained by correcting the chirped spectral phase of the pulses thanks to the natural dispersion of a metallic foil. The possibility of correcting the spectral phase with inversely chirped multilayer mirrors $[9,10]$ or with gratings compressors has also been demonstrated [11].

But, indeed, the phase difference between spectral components of an ultrashort pulse varies in space. For that reason, the pulse shape and the pulse duration may not be the same at every point in space, especially when focusing pulses with aberrating optics. Generally in order to manipulate attosecond pulses, on- and off-axis parabolic and spherical mirrors are used $[5,12]$.

Consequently, they introduce spherical aberration, coma, and astigmatism which could have a dramatic impact on the pulse duration. Indeed a 100 as pulse is $30 \mathrm{~nm}$ thick; that is to say a variation greater than $30 \mathrm{~nm}$ of the optical paths is sufficient to significantly stretch the attosecond pulse. The influence of aberrations on ultrashort pulses has already been studied for femtosecond pulses not only theoretically [13-16] but also experimentally [17]. But since optics used for visible femtosecond pulses is usually on-axis optics, spherical aberration and chromatism have been studied [15].

Moreover, currently there is no detector able to spatiotemporally characterize attosecond pulses. Indeed, to measure the pulse durations, current techniques are implementing time of flight (TOF) spectrometers, detecting the electrons coming from the ionization of a rare gas jet in which the pulses are focused $[5,12]$. Since the interaction in the gas is not spatially resolved, the pulses cannot be spatiotemporally characterized. In other words, the effects of geometric aberrations on attosecond pulses can be very important even for usual conjugations and, currently, their influence on the pulse duration is difficult to study experimentally.

In this paper, we propose a theoretical study of such effects. First of all, we describe the theoretical model used to perform the numerical simulations. Then we present the results obtained by using on- and off-axis parabolic and spherical multilayer mirrors, investigating the dependence of the pulse duration on the numerical aperture (NA).

\section{THEORETICAL MODEL}

We consider the optical layout presented in Fig. 1. In order to study the influence of geometric aberrations in a chosen configuration, we developed our own ray-tracing software. But around the focal point, it is not possible to describe attosecond pulses with geometric optics because of interferences and diffraction effects. Our solution is to use the ray tracing in order to describe the electric field in a deeply defocused plane, called integration plane in Fig. 1 , and where diffraction effects are negligible; then we calculate the attosecond pulse near the focal point by resolving the Rayleigh-Sommerfeld integral [18].

\section{A. Source}

The EUV electric field emitted by the source is spatially Gaussian [19]. Since the optics is always placed at a distance from the attosecond pulse source far bigger than the Rayleigh range for all the energies of the spectrum, we can consider that the intensity distribution on the mirror is a Gaussian angular distribution generated by a source point $S$ (see Fig. 1). Consequently the intensity distribution can be described by Eq. (1) as a function of the posi- 


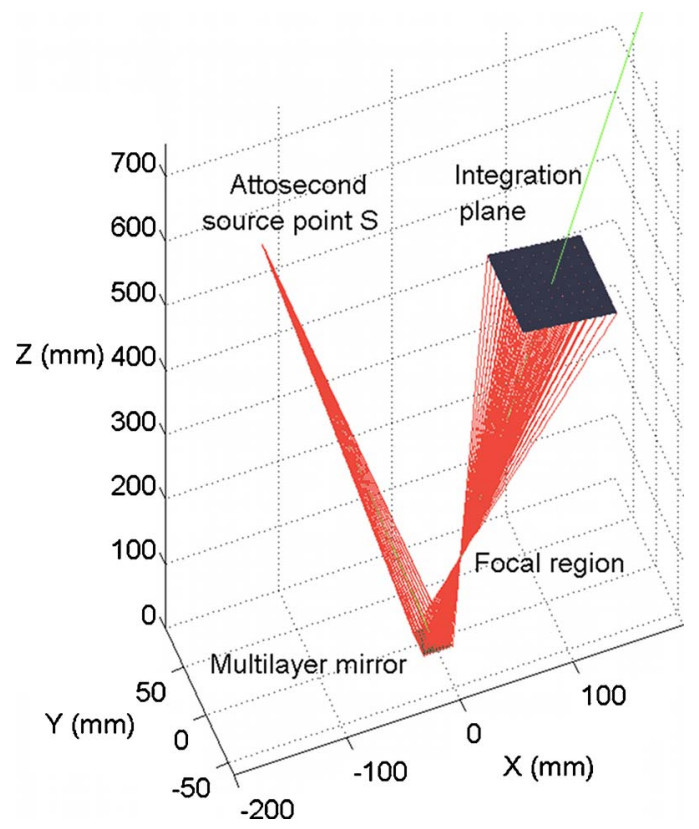

Fig. 1. (Color online) Reflection of the EUV beam on a mirror. The ray-tracing software allows us to geometrically describe the attosecond pulse in the integration plane.

tion $z$ along the beam propagation axis, the angle $\theta$ with this axis, and the wavelength $\lambda$,

$$
I(\theta, z, \lambda) \approx I_{0}(\lambda) \frac{w_{0}^{2}}{z^{2} \theta_{0}(\lambda)^{2}} \exp \left[\frac{-\theta^{2}}{2 \theta_{0}(\lambda)^{2}}\right],
$$

where $\theta_{0}(\lambda)$ is the divergence of the EUV beam and is equal to $\lambda / w_{0} \pi$, with $w_{0}$ being the beam waist, and $I_{0}(\lambda)$ is the EUV spectrum of the source.

\section{B. Mirror}

Let $M\left(x_{\text {mirror }}, y_{\text {mirror }}, z_{\text {mirror }}\right)$ be the current point on the mirror surface. The mirror has a given shape and a given coating, the complex reflectivity of which (reflected amplitude and reflected phase), called $r(S, M, \lambda)$, depends on the energy and on the angle of incidence. The reflectivity also depends on polarization, but in this work we only consider one component of the electric field, which is assumed to be one of the eigenpolarizations of the reflectivity. Each ray traced from the source to the mirror is assigned an elementary area on the mirror.

Knowing the local normal to the mirror, we can determine the solid angle $\Delta \Omega(M)$ under which that area is seen from the source. It allows us to calculate the flux $F_{r}(M, \lambda)$ on the mirror surface [see Eq. (2)],

$$
F_{r}(M, \lambda)=I(S, M, \lambda) \Delta \Omega(M)|r(S, M, \lambda)|^{2} .
$$

The ray tracing allows us to know the coordinates of the impact point $P\left(x_{\text {plane }}, y_{\text {plane }}, z_{\text {plane }}\right)$ of the reflected ray at point $M$ with the integration plane. Then we obtain the scalar electric field $E(P, \lambda)$ in this plane [see Eq. (3)], (a)

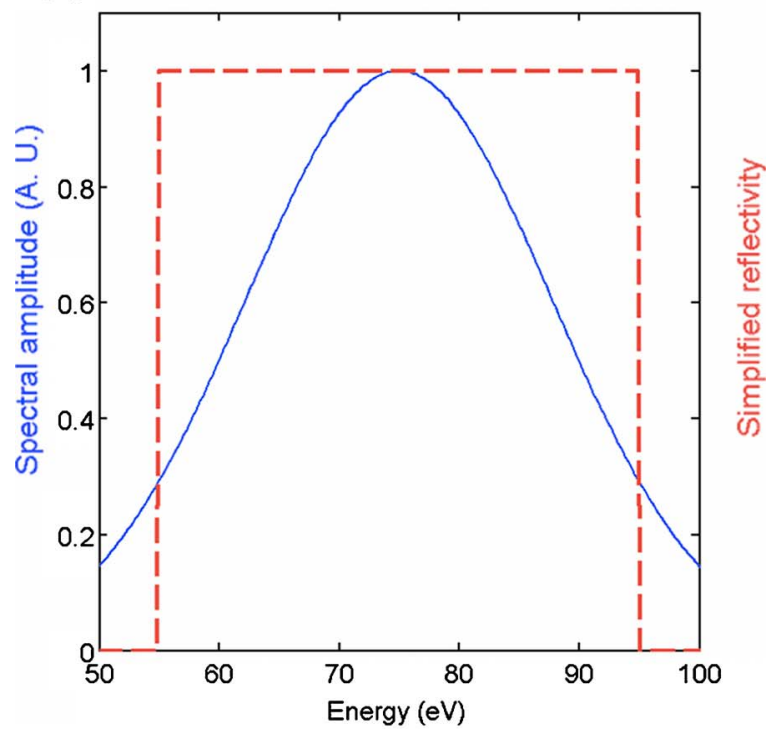

(b)

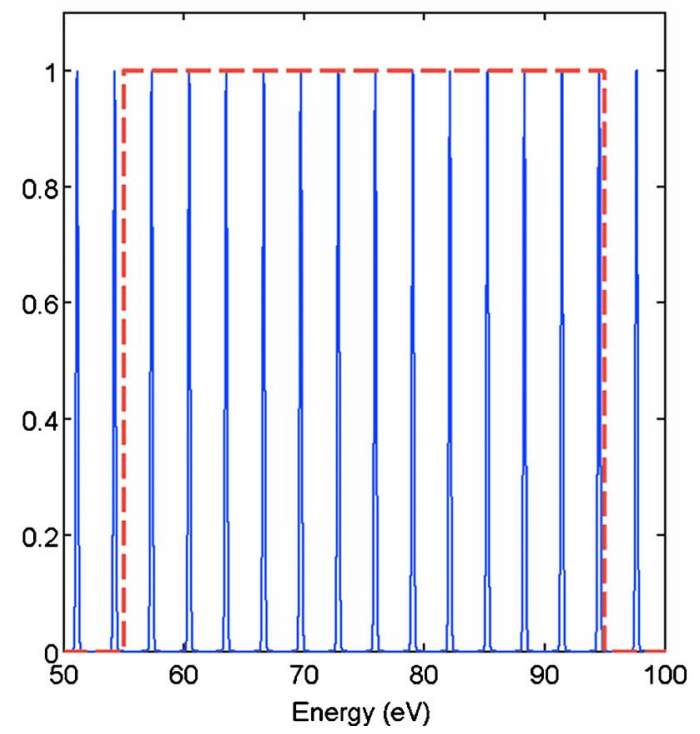

Fig. 2. (Color online) Spectral amplitude (a) of a single attosecond pulse and (b) of an attosecond pulse train. The reflectivity spectrum of the mirror is shown in dashed line.

$$
\begin{aligned}
E(P, \lambda)= & {\left[F_{r}(M, \lambda)\right]^{1 / 2} \exp \left\{i \phi_{\text {source }}(\lambda)+i \phi_{\text {mirror }}(P, \lambda)\right.} \\
& \left.+\frac{2 i \pi}{\lambda}[(S M)+(M P)]\right\},
\end{aligned}
$$

where $\phi_{\text {source }}(\lambda)$ is the spectral phase of the EUV electric field emitted by the source point $S, \phi_{\text {mirror }}(P, \lambda)$ stands for the phase reflected by the mirror, and $(S M)+(M P)$ represents the geometric optical path of the ray reflected at point $M$ and impacting at point $P$ on the integration plane.

\section{Detector}

In order to simulate the electric field of the pulse nearby the focal point $P^{\prime}\left(x_{\text {detector }}, y_{\text {detector }}, z_{\text {detector }}\right)$, we calculate the Rayleigh-Sommerfeld integral of the electric field in 


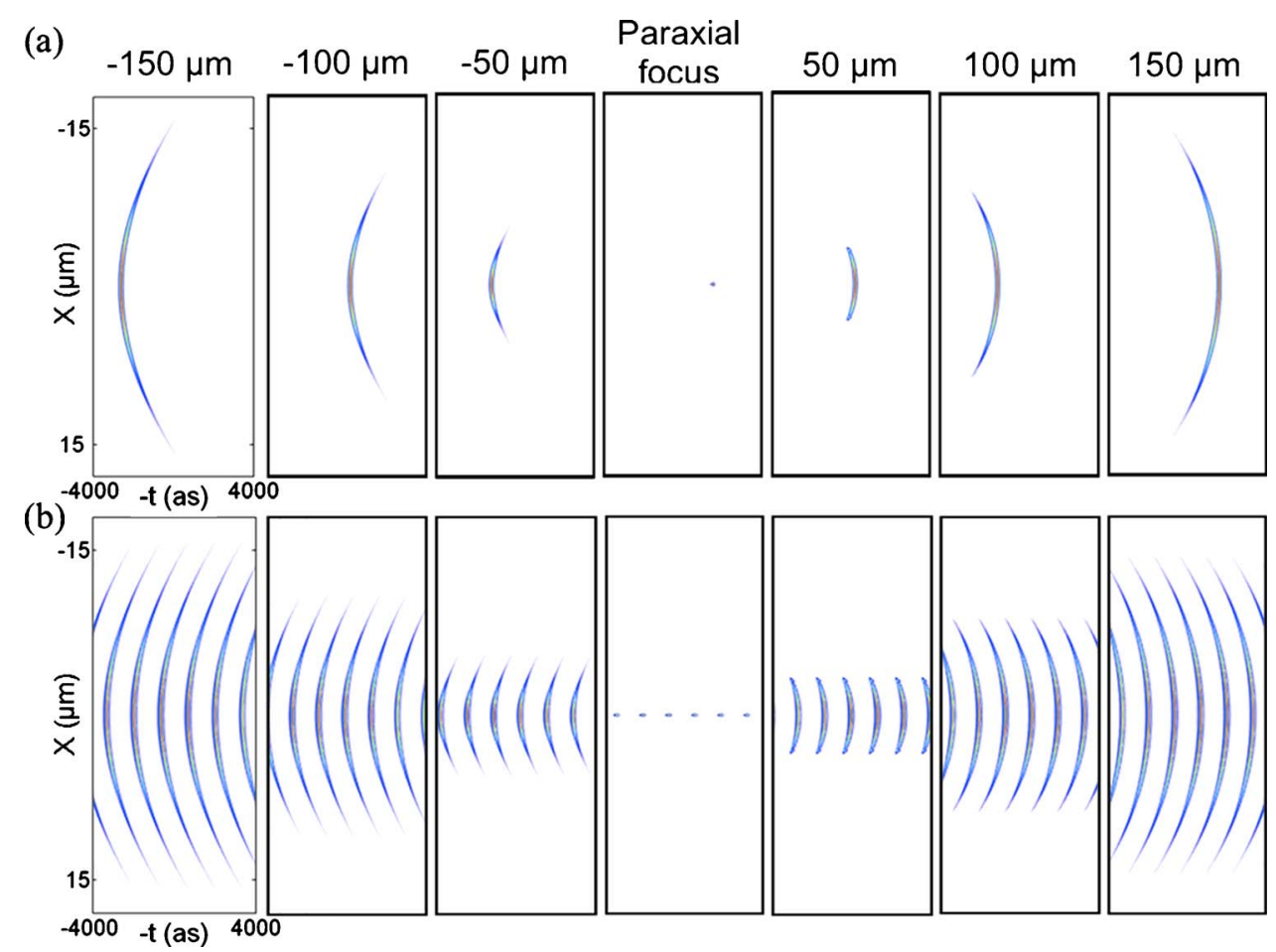

Fig. 3. (Color online) Radial intensity distribution of (a) a single attosecond pulse and (b) an attosecond pulse train focused by an onaxis parabolic mirror.

the integration plane $E(P, \lambda)$. And finally Eq. (4) gives us the electric field nearby the focal point,

$E\left(P^{\prime}, \lambda\right) \propto$

$$
-\frac{i}{\lambda} \int_{-\infty}^{+\infty} \int_{-\infty}^{+\infty} E(P, \lambda) \frac{\exp \left(\frac{2 i \pi}{\lambda} P P^{\prime}\right)}{P P^{\prime}} \cos \left[\gamma\left(P, P^{\prime}\right)\right] d S_{\text {plane }}
$$

where $\gamma\left(P, P^{\prime}\right)$ is the angle between the propagation axis and the $P P^{\prime}$ line. Note that $P P^{\prime}$ is an algebraic distance which becomes negative when $P$ is placed beyond $P^{\prime}$ in the way of the propagation of the beam. We can consider

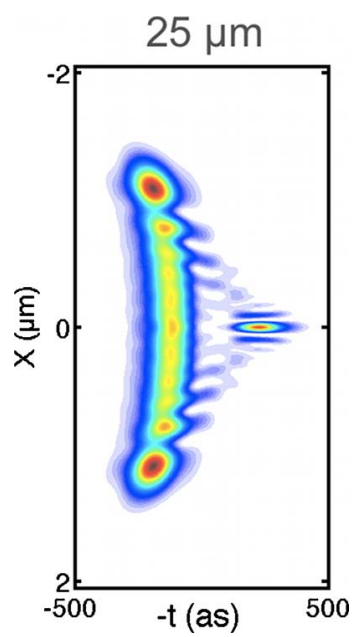

Fig. 4. (Color online) Radial intensity distribution of a single attosecond pulse focused by an on-axis parabolic mirror $25 \mu \mathrm{m}$ after the paraxial focus. that $\cos \left[\gamma\left(P, P^{\prime}\right)\right]$ is equal to 1 when the scalar approximation is verified. Finally we calculate the square modulus of the inverse Fourier transform of the previous electric field in order to obtain the spatiotemporal intensity distribution of the pulse [see Eq. (5)],

$$
I\left(P^{\prime}, t\right)=\left|F T^{-1}\left[E\left(P^{\prime}, v\right)\right]\right|^{2},
$$

where $v$ is the optical frequency and is equal to $c / \lambda$.

\section{CONDITIONS OF THE SIMULATIONS}

\section{A. Source}

The EUV radiation is generated by the interaction between an infrared femtosecond pulse and a neon gas jet. The EUV typical spectral amplitude is

- either Gaussian centered at $75 \mathrm{eV}$ with a full width at half-maximum (FWHM) of $30 \mathrm{eV}$, when a single attosecond pulse is emitted [8] [see Fig. 2(a)],

- or a high harmonic spectral amplitude, when an attosecond pulse train is generated [see Fig. 2(b)]. In this case, since high harmonics are generated in a rare gas jet, only odd harmonics are emitted. Moreover, considering the initial femtosecond pulse is centered at $800 \mathrm{~nm}$, the interval between two consecutive pulses of the train is about 1.3 fs [5].

Moreover, since we are considering short trajectories, attosecond pulses are positively chirped [6] and their group delay dispersion is about $3000 \mathrm{as}^{2} / \mathrm{rad}$, which is a typical value.

\section{B. Mirror}

In order to efficiently reflect attosecond pulses, we need to deposit a multilayer stack on the mirror, the reflected 


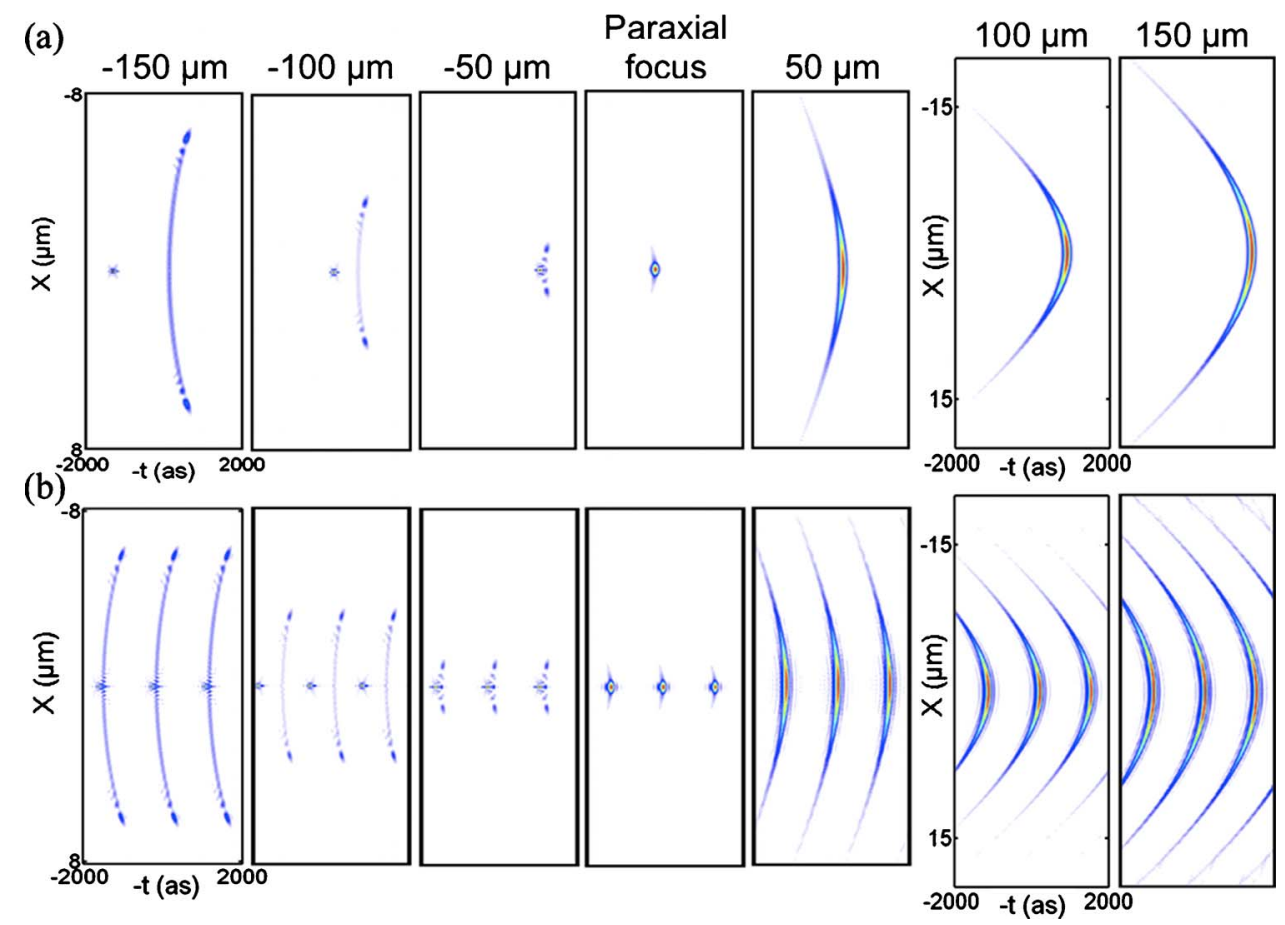

Fig. 5. (Color online) Radial intensity distribution nearby the paraxial focus when using an on-axis spherical mirror. We consider (a) a single attosecond pulse and (b) an attosecond pulse train.

spectral phase of which has to be as linear as possible over a large bandwidth with a constant reflectivity. Such multilayer mirrors exist near normal incidence and for the $35-75 \mathrm{eV}$ spectral range, as described in Fig. 5 of [20]. But the reflected phase of this mirror is not perfectly linear over the whole bandwidth so that such a stack may weakly stretch the reflected pulse. In the case of [20], we estimate such a stretch to a few tens of percents on the FWHM of the pulse.

But the point of this article being only to study the effects of geometric aberrations, we consider that the mirror has an ideal reflectivity which is constant between 55 and $95 \mathrm{eV}$ and null everywhere else. Moreover the reflected phase is linear on the whole bandwidth, with the result that the mirror will not modify the initial chirp of the pulse. In the following situations, the angles of incidence of the rays on the mirror will always be lower than $4^{\circ}$. Consequently it is reasonable to consider that the reflectivity and the reflected phase of the mirror will not change on this angular range [21].

\section{Detector}

In order to simulate the evolution of the pulse in the whole photoelectron collection volume in the TOF, typically $300 \mu \mathrm{m}$ long area, we calculate the spatiotemporal profile of the pulses every $50 \mu \mathrm{m}$ over a $300 \mu \mathrm{m}$ range centered on the paraxial focus.

\section{RESULTS}

\section{A. On-Axis Parabolic Mirror}

Let us consider an on-axis parabolic mirror with a focal length equal to $100 \mathrm{~mm}$. The mirror is placed $3 \mathrm{~m}$ behind the source and refocuses the beam into the rare gas jet of the TOF. The FWHM of the polychromatic Gaussian EUV spot on the mirror is about $8.4 \mathrm{~mm}$. Since the problem has a symmetry of revolution, calculating the radial intensity distribution in a plane is sufficient to perfectly know the whole pulse. In such a conjugation, a parabolic mirror is not very aberrating and the pulses are almost diffraction limited. Indeed we can clearly see in Fig. 3 that the pulse fronts are spherical.

But if we wanted to have a perfectly stigmatic conjugation, we would have to put the source at infinity, which is not rigorously the case. Indeed there is a small spherical aberration which is responsible for the intensity fluctuations visible on the boundaries of the pulse fronts $50 \mu \mathrm{m}$ after the paraxial focus. The simulation of the pulse $25 \mu \mathrm{m}$ after the paraxial focus highlights such an influence of spherical aberration (see Fig. 4). Paraxial rays which have already been focused at the paraxial focus are responsible for the main diverging part of the pulse. But the second part of the pulse which is propagating before the first one is generated by marginal rays coming from the boundaries of the mirror. Indeed their optical paths are shorter than the paraxial rays', with the result that the marginal part of the pulse is ahead of the paraxial one.

\section{B. On-Axis Spherical Mirror}

Let us replace the previous parabolic mirror with a spherical one. This conjugation is not stigmatic since the mirror adds a significant spherical aberration (see Fig. 5). Whereas spherical aberration was very small when using a parabolic mirror, this time the influence of spherical aberration is very important no matter what is the position in the rare gas jet. Especially $150 \mu \mathrm{m}$ before the paraxial focus, the pulse is already composed of two main parts due to the different focal points for paraxial and marginal rays. Such a pulse usually called a Bessel-like pulse is a 

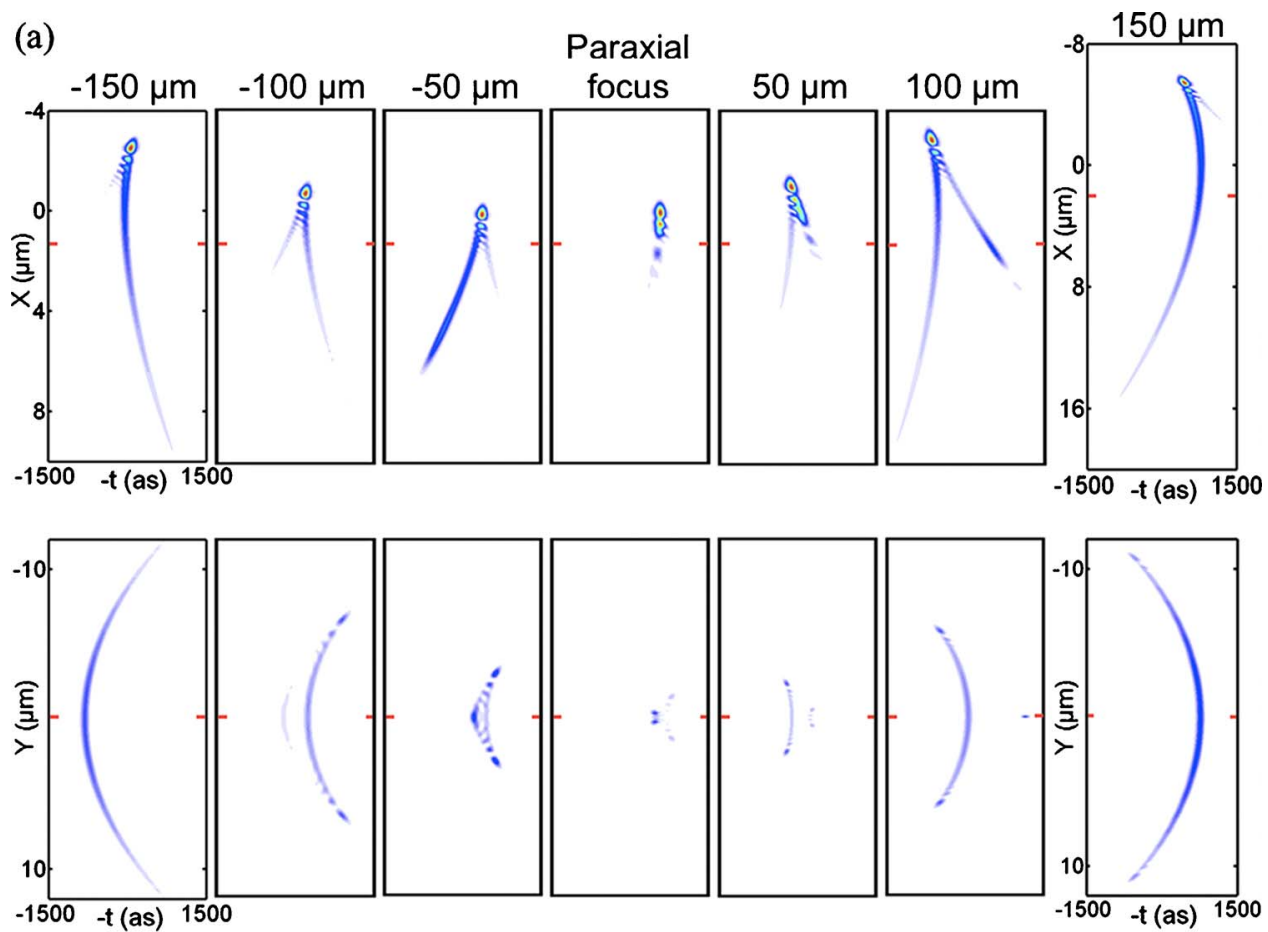

(b)
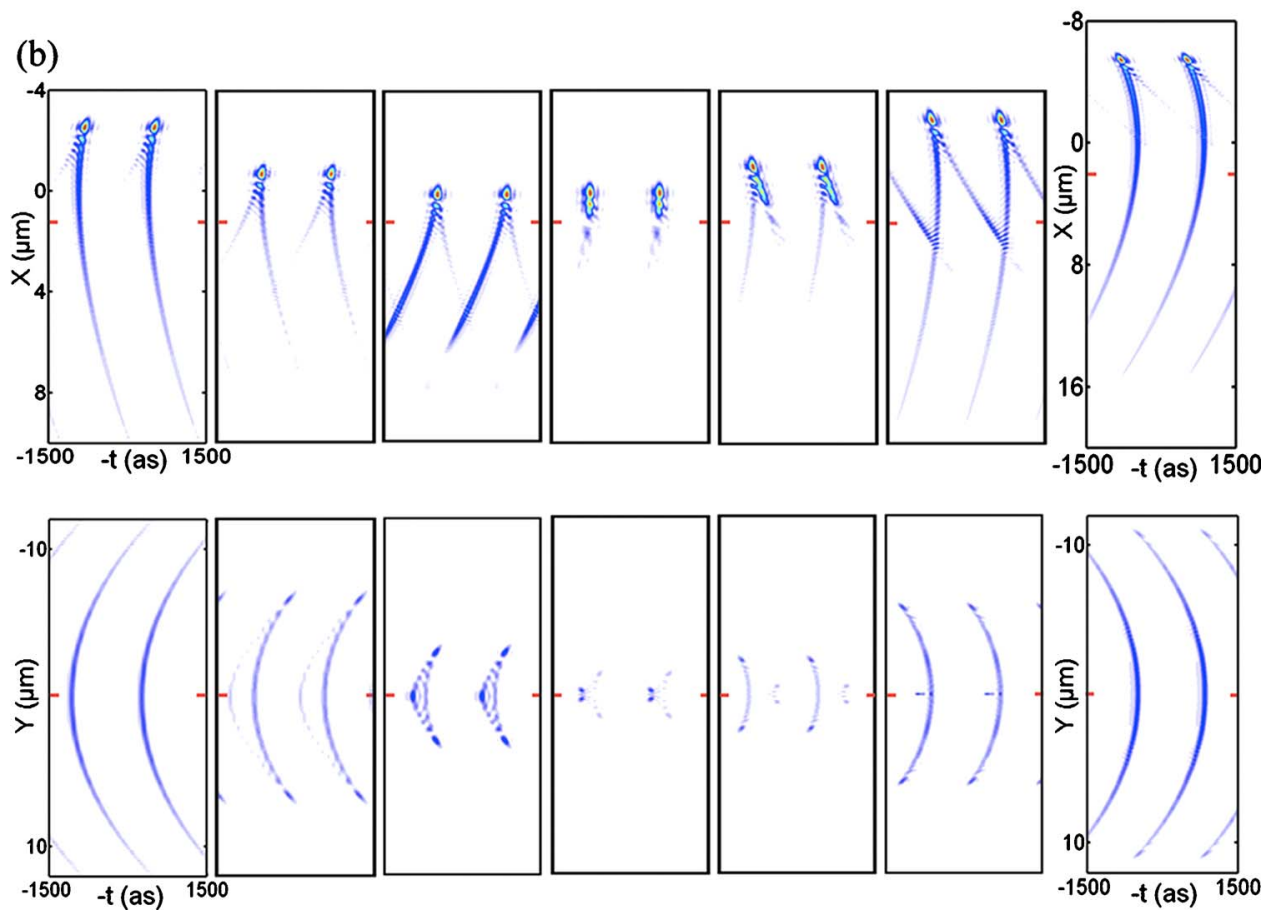

Fig. 6. (Color online) Intensity distribution in two orthogonal planes when studying either (a) a single attosecond pulse or (b) an attosecond pulse train after their reflection on a $1^{\circ}$ field angle parabolic mirror. The bold lateral lines on each image indicate the position of the corresponding orthogonal section of the pulse.

typical effect of spherical aberration on ultrashort pulses and is quite well-known with visible femtosecond pulses [15-17].

Concerning the reflection of an attosecond pulse train, with our realistic assumption of pulses spaced by $1.3 \mathrm{fs}$, this effect is so important that the paraxial part of one pulse over-passes the marginal part of the previous one. Indeed we can see interferences between two consecutive pulses in Fig. 5(b). These interferences disappear $50 \mu \mathrm{m}$ later since the marginal and paraxial parts of each attosecond pulse of the train come closer. At the paraxial focus, the beam size is the smallest but the lateral part of the pulse is slightly delayed with respect to the on-axis part of the pulse. After the paraxial focus, the pulse front is quickly diverging without generating any interference effects between different attosecond pulses of the train. This clearly means that interference effects appear if the pulse duration at a given point exceeds $1.3 \mathrm{fs}$. 
(a)
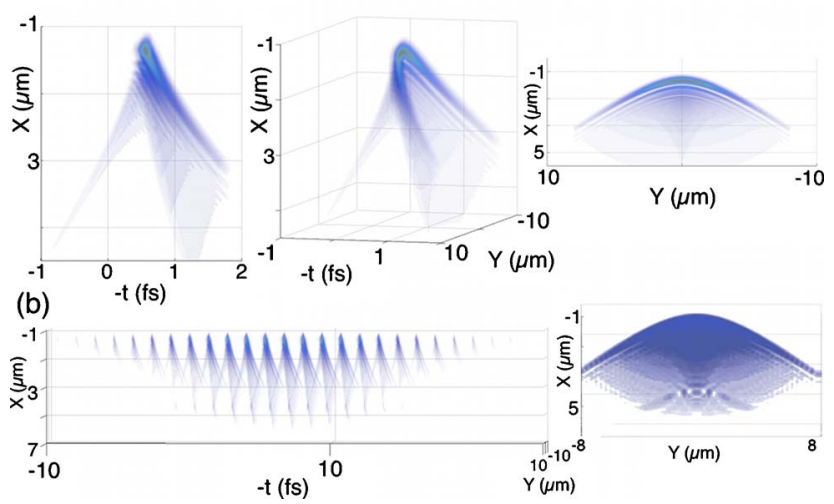

Fig. 7. (Color online) 3D Intensity distribution (a) of a single attosecond pulse or (b) of an attosecond pulse train $75 \mu \mathrm{m}$ before the paraxial focus after their reflection on a $1^{\circ}$ field angle parabolic multilayer mirror.

\section{Off-Axis Parabolic Mirror}

Let us now study off-axis mirrors. We consider a $1^{\circ}$ field angle parabolic mirror. This time, in addition to a small spherical aberration, we now add a significant coma aberration and a very small astigmatism. Since the problem does not have any symmetry of revolution anymore, we have to study the pulses in two orthogonal planes in order to properly describe them. First of all, we can see in Fig. 6 that the effect of coma on pulses is a far cry from spherical aberration. Indeed coma generates V-shaped pulses made of two parts which join together to create the most intense part of the pulse [16].

When considering an attosecond pulse train, we can see interference effects between two consecutive pulses at several positions, especially between -100 and $-50 \mu \mathrm{m}$. Consequently we simulate at $-75 \mu \mathrm{m}$ the pulses not only in two orthogonal planes but also in the whole volume in order to resolve the complete fringe system. In Fig. 7(b), we can see on the side view some dark spots at $X$ $=4.3 \mu \mathrm{m}$ where two consecutive pulses are interfering. On the front view, we see that the fringes are well contrasted and are located on a bow.

\section{Off-Axis Spherical Mirror}

In our last case, we replace the previous parabolic mirror with the spherical one with the result that we still have a lot of comas, but we add a significant spherical aberration. Consequently, calculations show pulses which are quite similar to those obtained when considering an onaxis spherical mirror, especially for the $Y$ sections (see Fig. 8). But the influence of coma is still important and is clearly visible on the $X$ sections since coma removes the symmetry of revolution of the pulse by making it have a $\mathrm{V}$ shape.

Concerning interferences between consecutive pulses, we can see that they are particularly important in this configuration $150 \mu \mathrm{m}$ before the paraxial focus. Indeed, Fig. 8(b) shows us at least two fringe systems. In order to study more efficiently these interferences, we perform the same kind of calculations as previously. We calculate the three-dimensional (3D) intensity distribution at this position. On the side view of Fig. 9(b), we can see in fact four series of aligned dark spots which are standing for the four fringe systems. This conclusion is confirmed in Fig. 10 which shows that the four fringe systems are equally spaced by about $3 \mu \mathrm{m}$. Moreover we can see on the front view of Fig. 9(b) that these interferences are located on bows. What is remarkable on these fringe systems is that they are all corresponding to different kinds of interferences. More precisely, they are always generated by interferences between two pulses but these two pulses are varying depending on the fringe systems. In fact, starting from the top of Fig. 10:

- the first system corresponds to interferences between two consecutive pulses,

- the second one corresponds to interferences between two pulses spaced by one pulse in the train,

- the third one corresponds to interferences between two pulses spaced by two pulses in the train, and

- finally the fourth fringe system corresponds to interferences between two pulses spaced by three pulses in the train.

\section{E. Influence of the Numerical Aperture on the Pulse Duration}

When studying geometric aberrations, the key parameter is the image NA. A small increase in this quantity can significantly increase the influence of aberrations. That is why it is interesting to study the evolution of aberrations, and especially of the pulse duration, with respect to the image NA.

In order to define a pulse duration based on our 3D intensity distribution, we measure the duration of the spatially integrated 3D pulse at the paraxial focus, just as if we were measuring the pulse duration on the signal of an imaginary attosecond photodiode. In the four previous cases, we calculate the FWHM and the $80 \%$ energy pulse duration. Results are shown in Fig. 11.

As predicted, the influence of geometric aberrations on the pulse duration is strongly depending on the image NA. But more precisely, we see that the stretch of the pulse is much more important on the $80 \%$ energy pulse duration than on the FWHM. Indeed, the aberrations usually add some strongly delayed and weakly intense parts on pulses. When we spatially integrate the pulse, these strongly delayed parts are best taken into account in the $80 \%$ energy pulse duration than on the FWHM.

Concerning the on-axis parabolic mirror, Fig. 11 shows that we can significantly stretch the pulse even when working in this almost stigmatic conjugation. Indeed the small spherical aberration can be strong enough for NA $=0.047$ to double the $80 \%$ energy pulse duration. Concerning the on-axis spherical mirror, spherical aberration is much more important than in the previous situation, with the result that the pulse duration increases much more quickly. This is due to marginal rays which are significantly delayed with respect to paraxial ones. These rays are responsible for the diverging lateral part of the pulse at the paraxial focus in Fig. 5(a).

Since spherical aberration does not depend on the field angle, the increase in the pulse durations when considering off-axis mirrors is mainly due to coma aberration. But such an increase in the pulse duration is much more important when considering the off-axis parabolic mirror 

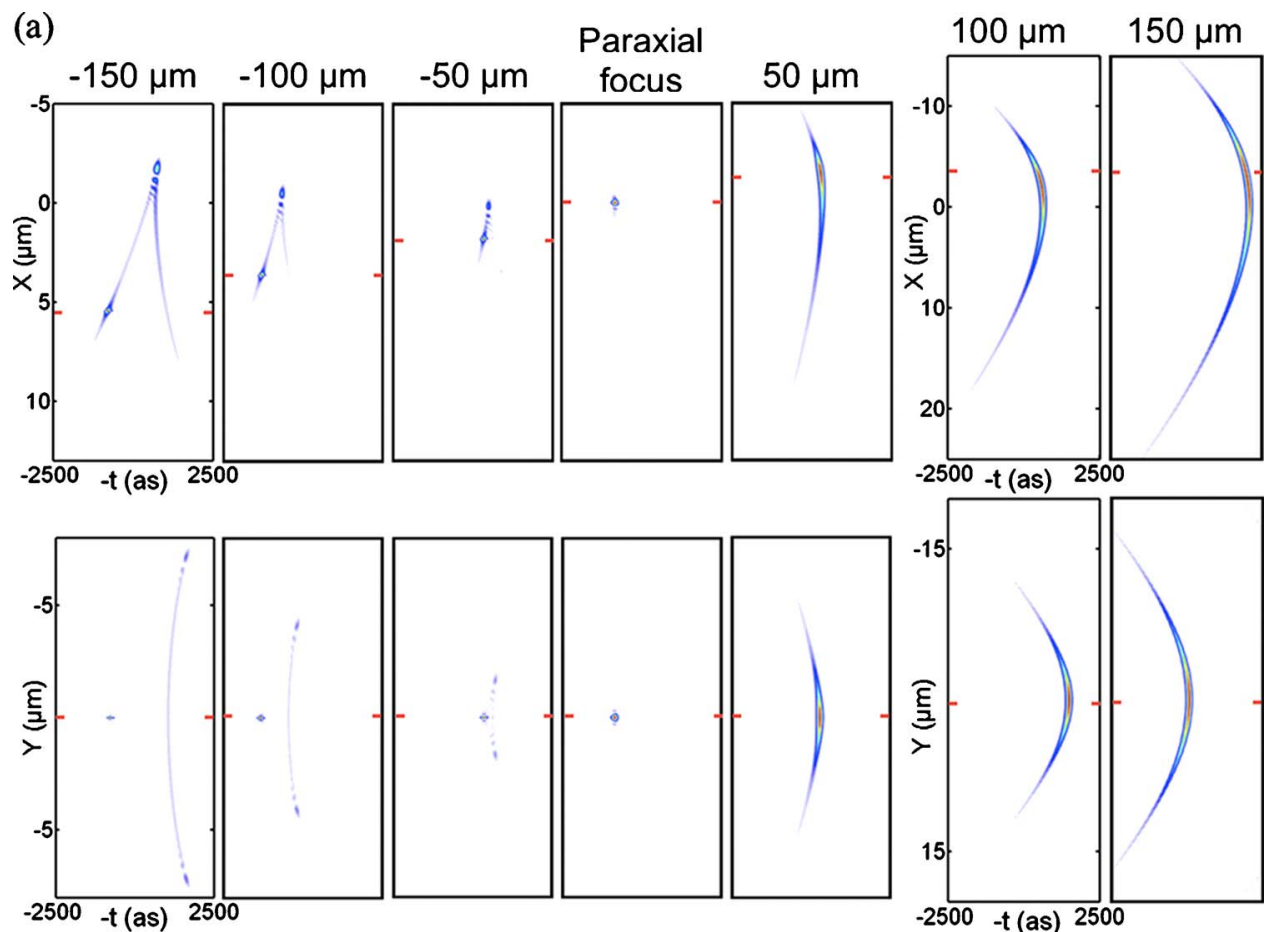

(b)
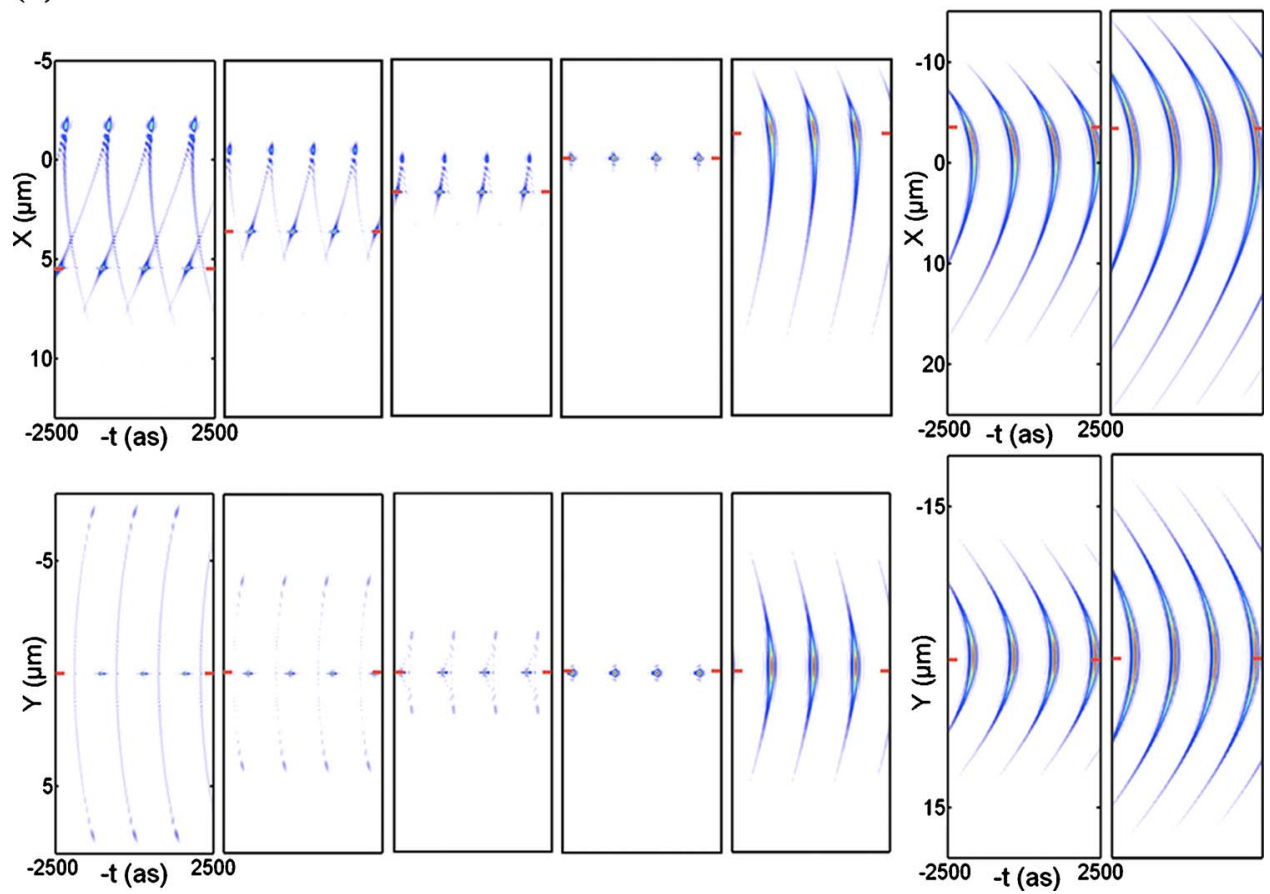

Fig. 8. (Color online) Intensity distribution in two orthogonal planes when studying either (a) a single attosecond pulse or (b) an attosecond pulse train after their reflection on a $1^{\circ}$ field angle spherical mirror.

than when considering the spherical one. This is clearly visible if we compare the two intensity distributions in Figs. 6(a) and 8(a). Indeed we can see that the pulse is more temporally stretched at the paraxial focus after its reflection on an off-axis parabolic mirror than on a spherical one.

Finally we can see that when considering the image $\mathrm{NA}$, the smaller is the better whatever is the case. Indeed we can see that even when using an off-axis parabolic mirror, the stretch can be negligible if the NA is lower than 0.015. But when considering such a low NA, a small stretch due to imperfections in the linearity of the spectral phase reflected by a real multilayer mirror may exceed aberrations' effects. Consequently, if we made calculations with real performances of the multilayer stack described in [20] instead of simplified performances (perfectly linear spectral phase and constant reflectivity) and for an initial spectrum centered at $50 \mathrm{eV}$, the normalized FWHM would have tended to 1.2 instead of 1 for low apertures. 


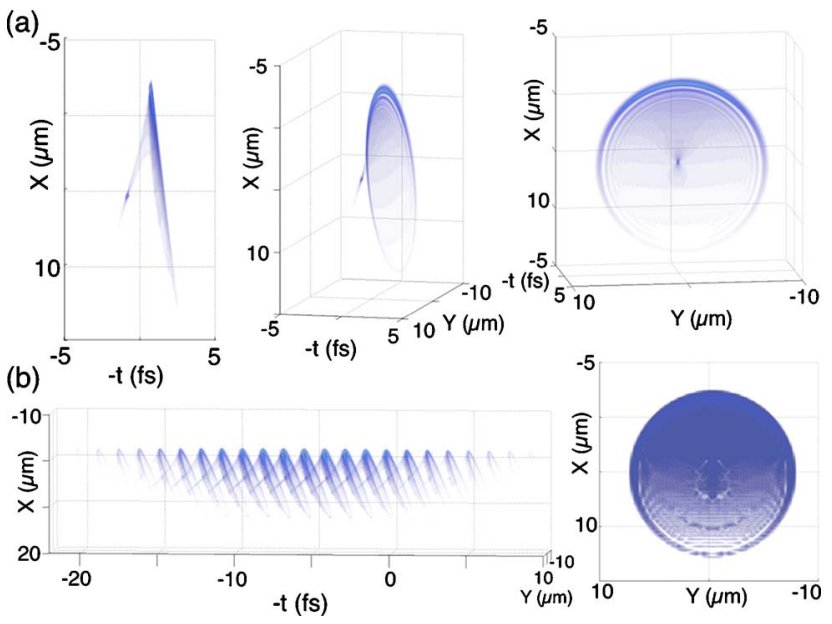

Fig. 9. (Color online) 3D Intensity distribution (a) of a single attosecond pulse or (b) of an attosecond pulse train $150 \mu \mathrm{m}$ before the paraxial focus after its reflection on a $1^{\circ}$ field angle parabolic multilayer mirror.

\section{CONCLUSION}

In conclusion, we developed a calculation code able to simulate the reflection of attosecond pulses on multilayer mirrors. We couple a ray-tracing code with a diffractive propagation code in order to take into account not only geometric effects but also diffraction effects. For the first time, to our knowledge, such a simulation code allowed one to study the influence of geometric aberrations on attosecond pulses.

Such an influence is a major issue, insofar as pulses can be strongly stretched by geometric aberrations even for usual configurations and small numerical apertures (NAs). Consequently the pulse duration can be significantly increased, and when considering attosecond pulse train, the superposition of several consecutive pulses of the train can generate multiple interference systems. Finally we demonstrated the strong dependence of the pulse duration on the NA, showing the latter should be lower

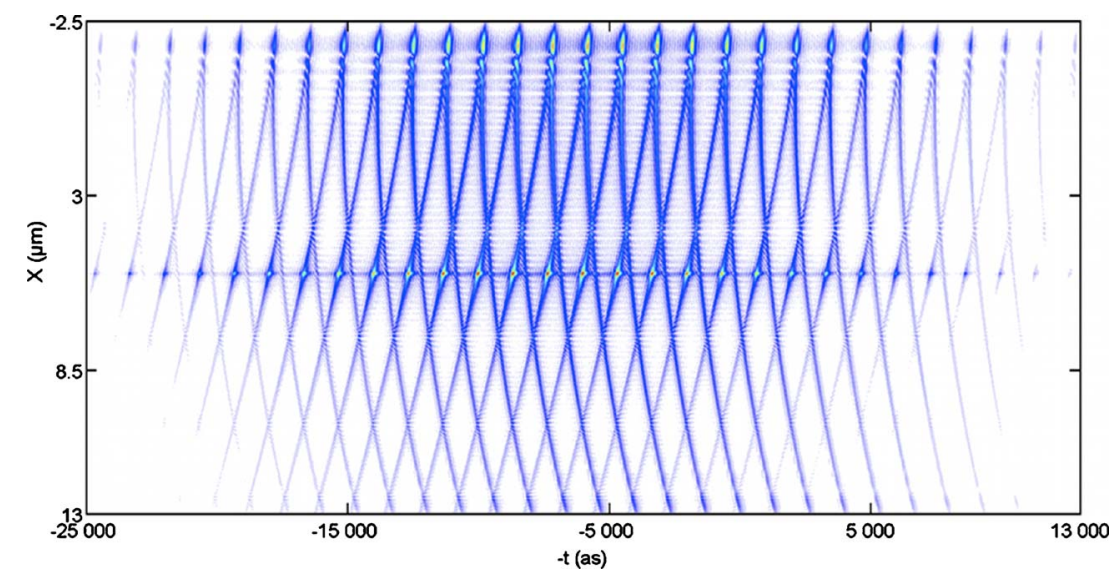

Fig. 10. (Color online) Modulus of the electric field in the plane of symmetry of the pulse train reflected by a $1^{\circ}$ field angle spherical mirror.

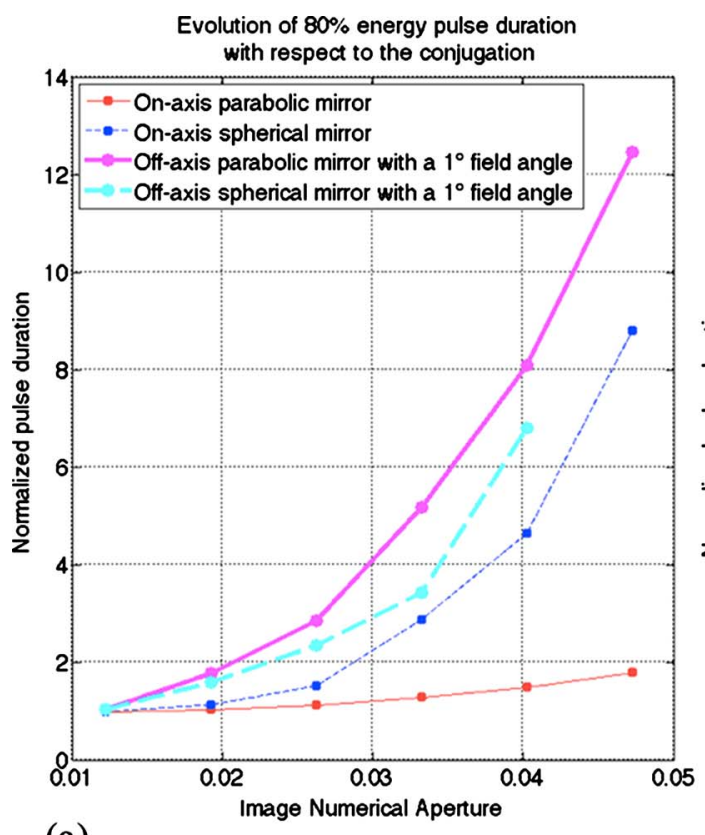

(a)

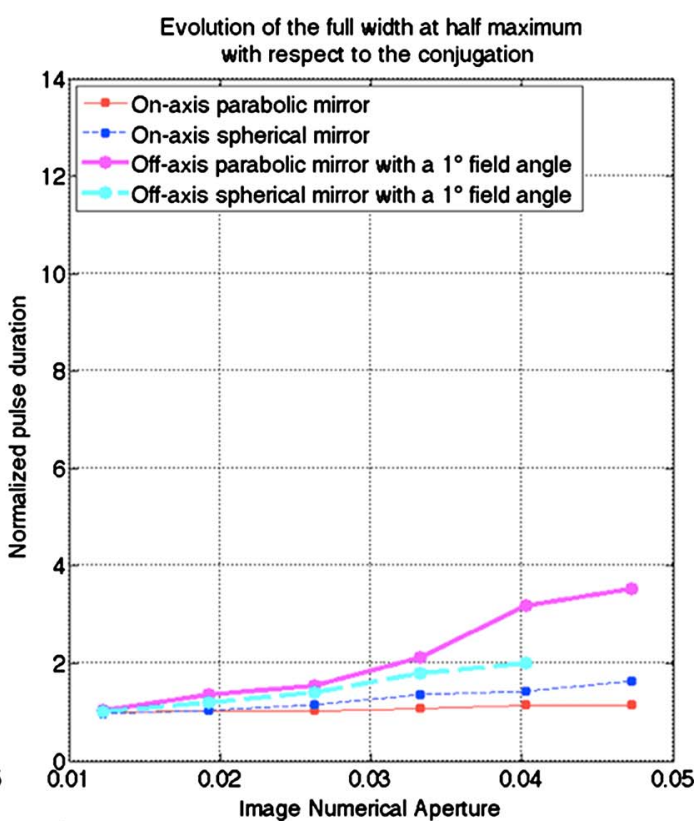

(b)

Fig. 11. (Color online) Evolution of (a) the 80\% energy pulse duration and (b) the FWHM with respect to the image NA. The pulse durations are normalized with respect to the initial pulse duration. 
than 0.015 to prevent aberration effects from stretching pulses for the cases investigated.

\section{ACKNOWLEDGMENT}

The authors would like to thank Raymond Mercier for fruitful discussions.

\section{REFERENCES}

1. A. McPherson, G. Gibson, H. Jara, U. Johann, T. S. Luk, I. A. McIntyre, K. Boyer, and C. K. Rhodes, "Studies of multiphoton production of vacuum-ultraviolet radiation in the rare gases," J. Opt. Soc. Am. B 4, 595-601 (1987).

2. M. Ferray, A. L'Huillier, X. F. Li, L. A. Lompre, G. Mainfray, and C. Manus, "Multiple-harmonic conversion of 1064 $\mathrm{nm}$ radiation in rare gases," J. Phys. B 21, L31-L35 (1988).

3. W. Boutu, S. Haessler, H. Merdji, P. Breger, G. Waters, M. Stankiewicz, L. J. Frasinski, R. Taieb, J. Caillat, A Maquet, P. Monchicourt, B. Carré, and P. Salières, "Coherent control of attosecond emission from aligned molecules," Nat. Phys. 4, 545-549 (2008).

4. F. Quéré, C. Thaury, P. Monot, S. Dobosz, Ph. Martin, J.-P. Geindre, and P. Audebert, "Coherent wake emission of high-order harmonics from overdense plasmas," Phys. Rev. Lett. 96, 125004 (2006).

5. P. M. Paul, E. S. Toma, P. Breger, G. Mullot, F. Augé, Ph. Balcou, H. G. Muller, and P. Agostini, "Observation of a train of attosecond pulses from high harmonic generation," Science 292, 1689-1692 (2001).

6. Y. Mairesse, A. de Bohan, L. J. Frasinski, H. Merdji, L. C. Dinu, P. Monchicourt, P. Breger, M. Kovacev, R. Taïeb, B. Carré, H. G. Muller, P. Agostini, and P. Salières, "Attosecond synchronization of high-harmonic soft x-rays," Science 302, 1540-1543 (2003).

7. M. Hentschel, R. Kienberger, C. Spielmann, G. A. Reider, N. Milosevic, T. Brabec, P. Corkum, U. Heinzmann, M. Drescher, and F. Krausz, "Attosecond metrology," Nature 414, 509-513 (2001).

8. E. Goulielmakis, M. Schultze, M. Hofstetter, V. S. Yakovlev, J. Gagnon, M. Uiberacker, A. L. Aquila, E. M. Gullikson, D. T. Attwood, R. Kienberger, F. Krausz, and U. Kleineberg,
"Single-cycle nonlinear optics," Science 320, 1614-1617 (2008).

9. A. S. Morlens, R. López-Martens, O. Boyko, P. Zeitoun, P. Balcou, K. Varjú, E. Gustafsson, T. Remetter, A. L’Huillier, S. Kazamias, J. Gautier, F. Delmotte, and M. F. Ravet, "Design and characterization of extreme-ultraviolet broadband mirrors for attosecond science," Opt. Lett. 31, 1558-1560 (2006).

10. A. Aquila, F. Salmassi, and E. Gullikson, "Metrologies for the phase characterization of attosecond extreme ultraviolet optics," Opt. Lett. 33, 455-457 (2008).

11. L. Poletto, F. Frassetto, and P. Villoresi, "Design of an extreme-ultraviolet attosecond compressor," J. Opt. Soc. Am. B 25, B133-B136 (2008).

12. M. Drescher, M. Hentschel, R. Kienberger, G. Tempea, C. Spielmann, G. Reider, P. Corkum, and F. Krausz, "X-ray pulses approaching the attosecond frontier," Science 291, 1923-1927 (2001).

13. Z. Bor, "Distortion of femtosecond laser pulses in lenses," Opt. Lett. 14, 119-121 (1989).

14. M. Kempe and W. Rudolph, "Femtosecond pulses in the focal region of lenses," Phys. Rev. A 48, 4721-4729 (1993).

15. U. Fuchs, U. D. Zeitner, and A. Tünnermann, "Ultra-short pulse propagation in complex optical systems," Opt. Express 13, 3852-3861 (2005).

16. Z. L. Horvàth, A. P. Kovács, and Z. Bor, "Distortion of ultrashort pulses caused by aberrations" in International Conference on Ultrafast Phenomena (UP), 2006 OSA Technical Digest Series (Optical Society of America, 2006), paper ThD16.

17. P. Bowlan, U. Fuchs, R. Trebino, and U. D. Zeitner, "Measuring the spatiotemporal electric field of tightly focused ultrashort pulses with sub-micron spatial resolution," Opt. Express 16, 13663-13675 (2008).

18. M. Born and E. Wolf, Principles of Optics, 7th ed. (Pergamon, 1999), pp. 514-515.

19. P. Salières, A. L'Huillier, and M. Lewenstein, "Coherence control of high order harmonics," Phys. Rev. Lett. 74, 37763779 (1995).

20. Y. Ménesguen, S. de Rossi, E. Meltchakov, and F. Delmotte, "Aperiodic multilayer mirrors for efficient broadband reflection in the extreme ultraviolet," Appl. Phys. A 98, 305-309 (2010).

21. L. G. Parratt, "Surfaces studies of solids by total reflection of x-rays," Phys. Rev. 95, 359-369 (1954). 